\title{
Factors Relating to the Abundance of Bacteria in the Mangrove Estuary of Nakama River, Iriomote Island, Okinawa
}

\author{
Hideo Miyoshi ${ }^{1}$, Souwapa Angsupanich ${ }^{2}$, and Yoshihiko Hata ${ }^{2}$ \\ 1. Usa Marine Biological Institute, Kochi University, Usa-cho, Tosa, Kochi 781-11, \\ Japan \\ 2. Faculty of Agriculture, Kochi University, Monobe, Nankoku, Kochi 783, Japan
}

\begin{abstract}
Physical, chemical and bacteriological examinations of water and mud from the mangrove estuary of Nakama River were carried out. The numbers of total bacteria in the water were one order of magnitude larger in summer than in winter, on the average, and had close correlations with a lot of variables such as, chlorinity, concentrations of nitrate, nitrite, ammonia, total nitrogen, particulate carbohydrates, particulate amino acids and chlorophyll $a$ in the water. The numbers of aerobic heterotrophic bacteria in the mud showed the same seasonal pattern as that for water bacteria, and had strong correlations with the concentrations of inorganic phosphate, ammonia and total nitrogen in the pore water as well as the contents of total carbohydrates and total amino acids in the mud. Interactions among water-related and mud-related variables were not so evident with few exceptions. The models for predicting bacterial abundance in the ecosystem are proposed by multivariate analysis, and the influence of environmental variables on the temporal and spatial distributions of bacteria in the estuary is discussed.
\end{abstract}

Key words : Bacterial distribution, multivariate analysis, Okinawa, mangrove estuary.

\section{Introduction}

Nakama River in Iriomote Island, Okinawa, has been totally undisturbed by pollutants resulting from human activity, and the middle estuary of the river has exuberant mangrove forest, which provides a source of organic matter.

Many workers have studied the relationships between bacterial populations or communities and biotic or abiotic variables in aquatic environments by multiple regression analysis (e.g., Mahloch, 1974 ; Miyoshi and Nakamoto, 1975 ; Jones, 1977 ; Palumbo and Ferguson, 1978; Väätänen, 1982 ; Hazen, 1983). The characteristics of bacterial community in Nakama River estuary were already reported in the previous number of this bulletin (Souwapa et al., 1989), but the influence of environmental variables on bacterial distribution in the mangrove estuary has not yet been elucidated.
We embarked on further study of physical and chemical variables relating to bacterial distribution in Nakama River estuary using multivariate analysis. The results obtained are reported herein.

\section{Study area}

Nakama River is located on the south-east coast of Iriomote Island (Fig. 1). The total length of the main stream is about $14 \mathrm{~km}$ and the daily tidal rise is observed up to Stn. 1. A small boat can pass upstream as far as $7.5 \mathrm{~km}$ at high tide, but only to Stn. 9 at neap tide, because the maximum tidal range is $1.2-2.5 \mathrm{~m}$ and the river is not very deep. Mangrove forest is most abundant in the middle estuary of the river where it covers about 300 hectares. 


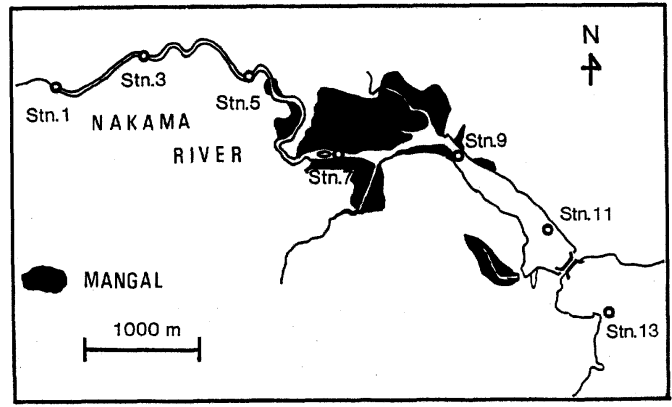

Fig. 1. Map showing the sampling stations in Nakama River.

\section{Materials and methods}

\section{Samples}

Water samples were collected with a liter bottle sampler at $1 \mathrm{~m}$ depth intervals throughout the entire water column. Particulate matter in the water was collected by filtration through precombusted glass fiber filters (Whatman GF/C). Mud was collected with a grab sampler and the uppermost layer $(c a .0-0.5 \mathrm{~cm})$ was used as the sample. Pore water was obtained from the mud sample by pressure filtration. Dry mud was prepared by heating at $105^{\circ} \mathrm{C}$ for $1 \mathrm{hr}$.

\section{Physical and chemical analyses}

Temperature and $\mathrm{pH}$ were measured immediately after sampling with a mercury-in-glass thermometer and a portable $\mathrm{pH}$ meter (Toa Electronics HM-10K), respectively. Analyses of nitrate, nitrite, ammonia, inorganic phosphate, total phosphorous, total nitrogen and chlorinity were carried out according to the methods described by Grasshoff et al. (1983). Particulate carbohydrates and total carbohydrates were determined by the phenol sulfuric acid method (Koyama et al., 1972). Particulate amino acids and total amino acids were determined by the ninhydrin method after being hydrolyzed with $6 \mathrm{~N} \mathrm{HCl}$ at $100^{\circ} \mathrm{C}$ for $24 \mathrm{hr}$. in a sealed tube (Koyama et al., 1982). Fluorometric determination of chlorophyll $a$ was performed on 90\% acetone extracts with a spectrofluoro- photometer (Shimadzu RF-500) (American Public Health Association, 1981).

\section{Counting of bacteria}

Epifluorescent counting of total bacteria in the water (water bacteria) was made using the technique described by Nishino (1986). Aerobic heterotrophic bacteria in the mud (mud bacteria) were counted according to the spread plate method (Buck and Cleverdon, 1960) with the following modifications: Media; Modified media of ZoBell's 2216E were employed. Namely, peptone and yeast extract were reduced to one tenth of original recipe, and for samples with low original salinities, the $80 \%$ strength seawater of original recipe was replaced by $33 \%$ strength seawater or tap water. Procedures; The salinity of pore water of each mud sample was roughly estimated using a refractory salinometer (Atago T-100). When the salinity of pore water was between $0-5$ $\% 0,5-15 \%$, or $>15 \%$, the following medium and diluent prepared respectively with tap water, $33 \%$ strength seawater or $80 \%$ strength seawater were employed, and spreading plates were made. After 2 weeks' incubation at $25^{\circ} \mathrm{C}$, the colonies developed on each plate were counted.

\section{Multivariate analysis}

Correlation and regression relationships were analyzed by using commercially available programs (Fujitsu “Analyst”).

\section{Results}

Physical, chemical and bacteriological examinations of water were carried out along a $7 \mathrm{~km}$ stretch of Nakama River estuary. Table 1 shows the results of these examinations. The numbers of water bacteria ranged from $c a .10^{4}$ to $10^{6}$ cells $\mathrm{ml}^{-1}$. In terms of seasonal variation, the numbers of water bacteria were approximately one order of magnitude larger in summer than in winter. Particulate carbohydrates, particulate amino acids and chlorophyll were also more abundant in 
summer. In addition, most levels of nitrogen components were slightly higher in summer. In terms of geographical distribution, chlorophyll, particulate carbohydrates and particulate amino acids were slightly abundant in the middle of estuary as compared with upper and lower parts of the stream, but the same pattern for the bacterial distribution was not evident.

The relationships among the variables related to the water are presented in Table 2. The log transformed counts of water bacteria had strong positive correlations with the concentrations of nitrate, nitrite, ammonia, total nitrogen, particulate carbohydrates, particulate amino acids, chlorophyll and temperature $(P<0.01)$, while the counts showed significant reverse correlation with chlorinity $(P<$ $0.01)$. Excluding bacterial variable, there are a large number of significant correlations among the remaining variables. In particular, temperature, the concentrations of ammonia, particulate amino acids and chlorophyll had close correlations with 8 or more of the 13 variables $(P<0.01)$.

The possible relationships between the bacterial variable (dependent variable) and a series of environmental variables (independent variables) were examined by the multiple regression analysis, and the following model for predicting bacterial population in the water $\left(\log Y_{w}\right.$, cells $\left.\bullet \mathrm{ml}^{-1}\right)$ is obtained :

$$
\begin{aligned}
\log Y_{w}= & -0.087 \mathrm{x}_{1}+0.035 \mathrm{x}_{2}-0.018 \mathrm{x}_{4} \\
& +0.117 \mathrm{x}_{5}-0.044 \mathrm{x}_{6}+0.967 \mathrm{x}_{8} \\
& +0.216 \mathrm{x}_{12}+4.867
\end{aligned}
$$

where $\mathrm{x}_{1}=\operatorname{depth}(\mathrm{m}), \mathrm{x}_{2}=$ temperature $\left({ }^{\circ} \mathrm{C}\right), \mathrm{x}_{4}=$ chlorinity $(\% 0), x_{5}=$ inorganic phosphate concentration $(\mu \mathrm{mol} / 1), x_{6}=$ total phosphorous concentration $(\mu \mathrm{mol} / 1), x_{8}=$ nitrite concentration $(\mu \mathrm{mol} /$ 1), $x_{12}=$ particulate amino acid concentration (as $\mathrm{mg}$ glutamic acid/1). The coefficient of determination $\left(R^{2}\right)$ of the model was 0.81 .

The results of physical, chemical and bacteriological examinations of mud and overlying water are presented in Table 3. The correlation coefficients between the variables are presented in
Table 4. The numbers of mud bacteria ranged from $\mathrm{ca} \cdot 10^{5}$ to $10^{7}$ colony forming units $(\mathrm{cfu}) / \mathrm{g}$ wet mud. The numbers were higher in summer than in winter. The concentrations of inorganic phosphate, ammonia and total nitrogen in the pore water were also higher in summer. The log transformed counts of mud bacteria had significant positive correlations with the concentrations of inorganic phosphate, ammonia, total nitrogen, total carbohydrates and total amino acids in the pore water or mud $(P<0.01)$.

Excluding the bacterial variable, there were 9 significant correlations among the remaining variables. In particular, the concentrations of inorganic phosphate and total nitrogen in the pore water and the contents of carbohydrates in the mud had close correlations with 3 variables besides bacterial variable $(P<0.01)$.

Based on the multiple regression analysis, the population of mud bacteria $\left(\log Y_{m}\right.$, cfu/g wet mud) can be predicted by the following model :

$$
\log Y_{m}=0.0385 \mathrm{x}^{\prime}{ }_{7}+0.9668
$$

where $\mathrm{x}^{\prime}{ }_{7}=$ total nitrogen concentration in the pore water $\left(\mu \mathrm{mol} \cdot 1^{-1}\right)$. The $R^{2}$ value of the model was 0.73 .

The relevant correlation coefficients, which were calculated by using the data shown in Tables 1, and 3, are given in Table 5. The result indicated that the log transformed counts of mud bacteria had significant correlations with the concentrations of ammonia, particulate amino acids and nitrite in the water $(P<0.05)$, while the $\log$ transformed counts of water bacteria had significant correlations with the concentrations of total nitrogen, inorganic phosphate and ammonia in the pore water $(P<0.05)$. Excluding bacterial variables, the concentrations of both ammonia and total nitrogen in the pore water had significant correlations with 4 or more out of the 9 variables related to water. 
Table 1. Data on the physical, chemical and bacteriological

\begin{tabular}{|c|c|c|c|c|c|c|c|c|}
\hline Date & Stn. & $\begin{array}{l}\text { Depth } \\
\text { (m) }\end{array}$ & $\begin{array}{l}\text { Temperature } \\
\left({ }^{\circ} \mathrm{C}\right)\end{array}$ & $\mathrm{pH}$ & $\begin{array}{c}\text { Chlorinity } \\
(\% 0)\end{array}$ & $\begin{array}{c}\text { Inorganic } \\
\text { phosphate } \\
(\mu \mathrm{mol} / l)\end{array}$ & $\begin{array}{c}\text { Total } \\
\text { phosphorus } \\
(\mu \mathrm{mol} / l)\end{array}$ & $\begin{array}{c}\text { Nitrate } \\
(\mu \mathrm{mol} / l)\end{array}$ \\
\hline $\begin{array}{l}\text { Feb 25, } \\
1987 \\
\text { (low tide) }\end{array}$ & $\begin{array}{r}3 \\
5 \\
7 \\
9 \\
11 \\
13\end{array}$ & $\begin{array}{l}0 \\
1 \\
0 \\
0 \\
0 \\
0 \\
1 \\
0 \\
1\end{array}$ & $\begin{array}{l}17.2 \\
17.8 \\
18.5 \\
18.8 \\
19.0 \\
18.5 \\
19.0 \\
19.0 \\
19.0\end{array}$ & $\begin{array}{l}6.9 \\
6.9 \\
6.9 \\
7.3 \\
7.7 \\
8.0 \\
8.0 \\
8.1 \\
8.1\end{array}$ & $\begin{array}{r}0.5 \\
2.4 \\
8.0 \\
12.0 \\
16.6 \\
18.5 \\
18.7 \\
18.9 \\
18.9 \\
\end{array}$ & $\begin{array}{l}0 \\
0 \\
0 \\
0.1 \\
0 \\
0 \\
0.1 \\
0.1 \\
0.1 \\
\end{array}$ & $\begin{array}{l}0.6 \\
0.5 \\
1.0 \\
0.6 \\
0.3 \\
0.3 \\
1.8 \\
0.5 \\
0.5 \\
\end{array}$ & $\begin{array}{l}2.6 \\
2.5 \\
2.7 \\
4.8 \\
3.1 \\
1.2 \\
0.8 \\
0.6 \\
0.4 \\
\end{array}$ \\
\hline $\begin{array}{l}\text { Feb 26, } \\
1987 \\
\text { (high tide) }\end{array}$ & $\begin{array}{l}1 \\
3 \\
5 \\
7 \\
7 \\
9 \\
11 \\
13\end{array}$ & $\begin{array}{l}0 \\
0 \\
1 \\
0 \\
1 \\
0 \\
1 \\
0 \\
1 \\
0 \\
1 \\
0 \\
1\end{array}$ & $\begin{array}{l}16.5 \\
17.5 \\
17.0 \\
17.3 \\
17.0 \\
17.0 \\
17.0 \\
17.0 \\
17.0 \\
17.5 \\
17.5 \\
18.2 \\
17.5 \\
\end{array}$ & $\begin{array}{l}7.1 \\
7.1 \\
7.2 \\
7.5 \\
7.5 \\
8.2 \\
8.2 \\
7.7 \\
8.2 \\
8.2 \\
8.2 \\
8.2 \\
8.2\end{array}$ & $\begin{array}{r}0.3 \\
0.7 \\
3.0 \\
8.4 \\
8.5 \\
17.5 \\
17.3 \\
18.2 \\
18.2 \\
18.7 \\
18.9 \\
19.4 \\
19.3\end{array}$ & $\begin{array}{l}0.1 \\
1.2 \\
1.2 \\
1.8 \\
1.7 \\
2.5 \\
3.0 \\
3.0 \\
2.0 \\
1.8 \\
1.7 \\
2.3 \\
1.6\end{array}$ & $\begin{array}{l}0.6 \\
1.6 \\
1.6 \\
1.9 \\
2.1 \\
3.0 \\
3.0 \\
3.3 \\
2.5 \\
2.5 \\
2.7 \\
2.5 \\
1.9\end{array}$ & $\begin{array}{l}3.0 \\
2.6 \\
2.8 \\
2.8 \\
1.4 \\
1.2 \\
1.2 \\
0.6 \\
1.2 \\
0.8 \\
0.5 \\
0.7 \\
0.4\end{array}$ \\
\hline $\begin{array}{l}\text { Aug 26, } \\
1987 \\
\text { (low tide) }\end{array}$ & $\begin{array}{r}3 \\
5 \\
5 \\
7 \\
9 \\
11 \\
13\end{array}$ & $\begin{array}{l}0 \\
1 \\
0 \\
1 \\
0 \\
0 \\
0 \\
0 \\
1\end{array}$ & $\begin{array}{l}30.0 \\
30.0 \\
32.0 \\
31.8 \\
32.0 \\
31.2 \\
31.3 \\
31.6 \\
32.0\end{array}$ & $\begin{array}{l}8.1 \\
8.0 \\
8.0 \\
8.0 \\
8.2 \\
8.0 \\
8.4 \\
8.5 \\
8.5\end{array}$ & $\begin{array}{r}2.4 \\
3.6 \\
4.0 \\
5.2 \\
9.6 \\
12.4 \\
15.0 \\
16.4 \\
16.5\end{array}$ & $\begin{array}{l}0.2 \\
0.2 \\
0 \\
0.2 \\
0.1 \\
0.1 \\
0.1 \\
0 \\
0.2\end{array}$ & $\begin{array}{l}1.2 \\
1.0 \\
0.8 \\
1.0 \\
1.2 \\
1.2 \\
1.8 \\
1.0 \\
1.1\end{array}$ & $\begin{array}{l}4.0 \\
3.6 \\
2.7 \\
2.6 \\
3.6 \\
3.0 \\
2.1 \\
1.3 \\
2.0\end{array}$ \\
\hline $\begin{array}{l}\text { Aug } 27 \\
1987 \\
\text { (high tide) }\end{array}$ & $\begin{array}{l}1 \\
3 \\
5 \\
7 \\
9\end{array}$ & $\begin{array}{l}0 \\
1 \\
0 \\
1 \\
0 \\
1 \\
0 \\
1 \\
0 \\
1 \\
3 \\
0 \\
1 \\
0 \\
1\end{array}$ & $\begin{array}{l}26.5 \\
29.5 \\
29.5 \\
30.0 \\
29.8 \\
29.8 \\
30.0 \\
30.0 \\
30.3 \\
30.0 \\
29.8 \\
30.0 \\
29.9 \\
30.0 \\
30.0\end{array}$ & $\begin{array}{l}8.2 \\
8.1 \\
8.3 \\
8.2 \\
8.4 \\
8.5 \\
8.5 \\
8.4 \\
8.5 \\
8.5 \\
8.5 \\
8.5 \\
8.5 \\
8.5 \\
8.5\end{array}$ & $\begin{array}{r}0.8 \\
2.5 \\
4.6 \\
5.4 \\
12.8 \\
14.4 \\
17.5 \\
18.3 \\
18.3 \\
18.3 \\
18.3 \\
18.5 \\
18.5 \\
19.0 \\
19.0\end{array}$ & $\begin{array}{l}2.2 \\
1.2 \\
1.2 \\
0.8 \\
1.0 \\
2.9 \\
4.5 \\
0.6 \\
0.4 \\
0.3 \\
0.4 \\
0.5 \\
0.2 \\
0.4 \\
0.4\end{array}$ & $\begin{array}{l}8.8 \\
8.5 \\
8.0 \\
4.1 \\
5.8 \\
6.0 \\
8.5 \\
2.5 \\
2.7 \\
4.1 \\
0.8 \\
3.0 \\
0.8 \\
2.3 \\
1.9\end{array}$ & $\begin{array}{l}4.7 \\
3.4 \\
3.8 \\
4.0 \\
3.4 \\
3.2 \\
0.7 \\
0.5 \\
0.5 \\
0.4 \\
0.4 \\
0.4 \\
0.5 \\
0.1 \\
0.2\end{array}$ \\
\hline
\end{tabular}


examinations of water from Nakama River estuary

\begin{tabular}{|c|c|c|c|c|c|c|}
\hline $\begin{array}{c}\text { Nitrite } \\
(\mu \mathrm{mol} / l)\end{array}$ & $\begin{array}{l}\text { Ammonia } \\
(\mu \mathrm{mol} / l)\end{array}$ & $\begin{array}{c}\text { Total } \\
\text { nitrogen } \\
(\mu \mathrm{mol} / l)\end{array}$ & $\begin{array}{c}\text { Particulate } \\
\text { carbohydrates } \\
\text { (as mg glucose } \\
/ l \text { ) }\end{array}$ & $\begin{array}{c}\text { Particulate } \\
\text { amino acids } \\
\text { (as mg glutamic } \\
\text { acid } / l \text { ) }\end{array}$ & $\begin{array}{c}\text { Chlorophyll } \\
a \\
\left(\mathrm{mg} / \mathrm{m}^{3}\right)\end{array}$ & $\begin{array}{c}\text { Water } \\
\text { bacteria } \\
(\text { cells } / \mathrm{ml})\end{array}$ \\
\hline 0.05 & 0.2 & 30.5 & 0.04 & 0.13 & 1.1 & $5.4 \times 10^{5}$ \\
\hline 0.05 & 0.3 & 31.0 & 0.08 & 0.36 & 3.0 & $2.3 \times 10^{5}$ \\
\hline 0.08 & 0.4 & 28.0 & 0.33 & 1.21 & 7.1 & $2.8 \times 10^{5}$ \\
\hline 0.05 & 0.2 & 27.0 & 0.11 & 0.51 & 1.6 & $4.1 \times 10^{5}$ \\
\hline 0.06 & 0.2 & 23.5 & 0.10 & 0.20 & 1.0 & $2.0 \times 10^{5}$ \\
\hline 0.03 & 0.1 & 22.0 & 0.06 & 0.14 & 0.8 & $1.7 \times 10^{5}$ \\
\hline 0.03 & 0.2 & 23.5 & 0.06 & 0.20 & 0.6 & $1.7 \times 10^{5}$ \\
\hline 0 & 0.1 & 22.0 & 0.07 & 0.15 & 0.9 & $2.0 \times 10^{5}$ \\
\hline 0 & 0.1 & 23.4 & 0.05 & 0.22 & 0.9 & $1.1 \times 10^{5}$ \\
\hline 0 & 0.7 & 23.5 & 0.03 & 0.11 & 1.2 & $6.3 \times 10^{4}$ \\
\hline 0 & 0.7 & 29.0 & 0.06 & 0.29 & 3.2 & $5.2 \times 10^{5}$ \\
\hline 0 & 0.6 & 29.0 & 0.13 & 0.51 & 1.4 & $4.7 \times 10^{5}$ \\
\hline 0 & 0.4 & 30.5 & 0.05 & 0.27 & 2.4 & $5.8 \times 10^{5}$ \\
\hline 0 & 0.4 & 30.0 & 0.12 & 0.43 & 1.6 & $5.4 \times 10^{5}$ \\
\hline 0 & 0.3 & 22.0 & 0.08 & 0.19 & 0.9 & $2.3 \times 10^{5}$ \\
\hline 0 & 0.3 & 34.5 & 0.04 & 0.23 & 1.0 & $2.0 \times 10^{5}$ \\
\hline 0 & 0.2 & 21.2 & 0.04 & 0.24 & 1.4 & $2.4 \times 10^{5}$ \\
\hline 0 & 0.2 & 22.0 & 0.06 & 0.26 & 0.7 & $1.5 \times 10^{5}$ \\
\hline 0 & 0.2 & 19.0 & 0.03 & 0.11 & 0.8 & $2.2 \times 10^{5}$ \\
\hline 0 & 0.3 & 27.5 & 0.02 & 0.16 & 0.7 & $1.8 \times 10^{5}$ \\
\hline 0 & 0.2 & 21.0 & 0.02 & 0.12 & 0.9 & $2.1 \times 10^{5}$ \\
\hline 0 & 0.4 & 19.1 & 0.02 & 0.14 & 0.9 & $1.1 \times 10^{5}$ \\
\hline 0.18 & 3.8 & 40.0 & 0.10 & 0.64 & 5.3 & $1.4 \times 10^{6}$ \\
\hline 0.19 & 3.1 & 37.0 & 0.22 & 0.89 & 6.2 & $1.3 \times 10^{6}$ \\
\hline 0.06 & 3.1 & 28.0 & 0.13 & 0.64 & 7.7 & $1.4 \times 10^{6}$ \\
\hline 0.25 & 2.7 & 36.0 & 0.15 & 0.78 & 6.3 & $1.6 \times 10^{6}$ \\
\hline 0.07 & 1.3 & 38.0 & 0.17 & 0.74 & 4.3 & $1.6 \times 10^{6}$ \\
\hline 0.23 & 1.0 & 35.0 & 0.17 & 0.71 & 3.2 & $1.1 \times 10^{6}$ \\
\hline 0.26 & 1.1 & 36.0 & 0.21 & 0.93 & 3.3 & $1.5 \times 10^{6}$ \\
\hline 0.21 & 0.3 & 22.5 & 0.15 & 0.55 & 2.5 & $1.0 \times 10^{6}$ \\
\hline 0.22 & 0.6 & 35.3 & 0.15 & 0.83 & 1.7 & $7.8 \times 10^{5}$ \\
\hline 0.11 & 2.7 & 48.0 & 0.05 & 0.22 & 1.4 & $6.1 \times 10^{5}$ \\
\hline 0.20 & 3.8 & 41.0 & 0.15 & 0.77 & 6.5 & $6.3 \times 10^{5}$ \\
\hline 0.23 & 2.4 & 68.0 & 0.14 & 0.73 & 7.4 & $1.2 \times 10^{6}$ \\
\hline 0.10 & 3.5 & 38.0 & 0.13 & 0.77 & 4.2 & $1.2 \times 10^{6}$ \\
\hline 0.20 & 2.7 & 31.0 & 0.08 & 0.49 & 2.6 & $1.2 \times 10^{6}$ \\
\hline 0.20 & 3.0 & 35.2 & 0.07 & 0.54 & 1.4 & $8.9 \times 10^{5}$ \\
\hline 0.10 & 1.4 & 35.2 & 0.09 & 0.64 & 4.1 & $6.1 \times 10^{5}$ \\
\hline 0.02 & 1.3 & 70.0 & 0.07 & 0.46 & 1.5 & $3.5 \times 10^{5}$ \\
\hline 0 & 0.8 & 31.0 & 0.09 & 0.42 & 2.8 & $5.1 \times 10^{5}$ \\
\hline 0 & 0.9 & 38.5 & 0.09 & 0.56 & 1.5 & $3.4 \times 10^{5}$ \\
\hline 0 & 1.4 & 28.0 & 0.07 & 0.49 & 1.3 & $3.5 \times 10^{5}$ \\
\hline 0 & 0.4 & 35.0 & 0.05 & 0.23 & 1.9 & $4.2 \times 10^{5}$ \\
\hline 0 & 0.5 & 35.7 & 0.08 & 0.37 & 1.0 & $2.7 \times 10^{5}$ \\
\hline 0.02 & 0.3 & 38.0 & 0.06 & 0.38 & 2.7 & $3.8 \times 10^{5}$ \\
\hline 0.02 & 0.6 & 61.0 & 0.06 & 0.40 & 1.6 & $2.6 \times 10^{5}$ \\
\hline
\end{tabular}


Table 2. Correlation matrix showing the degree of linear

\begin{tabular}{|c|c|c|c|c|c|c|}
\hline Variables & $\mathrm{x}_{1}$ & $\mathrm{x}_{2}$ & $x_{3}$ & $\mathrm{x}_{4}$ & $x_{5}$ & $\mathrm{x}_{6}$ \\
\hline Depth $\left(x_{1}\right)$ & 1.000 & & & & & \\
\hline Temperature $\left(x_{2}\right)$ & 0.093 & 1.000 & & & & \\
\hline $\mathrm{pH}\left(\mathrm{x}_{3}\right)$ & 0.191 & $0.641^{a}$ & 1.000 & & & \\
\hline Chlorinity $\left(\mathrm{x}_{4}\right)$ & 0.140 & -0.011 & $0.609^{a}$ & 1.000 & & \\
\hline Inorganic phosphate $\left(\mathrm{x}_{5}\right)$ & -0.024 & -0.242 & 0.168 & 0.168 & 1.000 & \\
\hline Total phosphorus $\left(\mathrm{x}_{6}\right)$ & -0.066 & 0.272 & 0.361 & -0.069 & $0.625^{a}$ & 1.000 \\
\hline Nitrate $\left(\mathrm{x}_{7}\right)$ & -0.237 & 0.130 & $-0.390^{a}$ & $-0.784^{a}$ & -0.141 & 0.182 \\
\hline Nitrite $\left(\mathrm{x}_{8}\right)$ & -0.105 & $0.615^{a}$ & 0.208 & -0.328 & -0.150 & 0.284 \\
\hline Ammonia $\left(x_{9}\right)$ & 0.105 & $0.613^{a}$ & 0.232 & $-0.529^{a}$ & 0.019 & $0.483^{a}$ \\
\hline Total nitrogen $\left(\mathrm{x}_{10}\right)$ & 0.108 & $0.569^{a}$ & 0.293 & -0.200 & -0.053 & $0.420^{a}$ \\
\hline Particulate carbohydrates $\left(\mathrm{x}_{11}\right)$ & -0.062 & $0.398^{a}$ & -0.123 & -0.320 & -0.360 & -0.072 \\
\hline Particulate amino acids $\left(\mathrm{x}_{12}\right)$ & 0.082 & $0.625^{a}$ & 0.074 & -0.319 & -0.238 & 0.126 \\
\hline Chlorophyll $\left(\mathrm{x}_{13}\right)$ & -0.159 & $0.491^{a}$ & 0.076 & $-0.564^{a}$ & -0.188 & 0.201 \\
\hline Total bacteria $\left(\log Y_{w}\right)$ & -0.144 & $0.715^{a}$ & 0.194 & $-0.428^{a}$ & -0.110 & 0.244 \\
\hline
\end{tabular}

$a$, significant at $0.01 \%$ level

\section{Discussion}

The numbers of water bacteria had close correla- tions with as many as 9 out of the 13 environmental variables related to water (Table 2). Especially, tight couplings were observed among the

Table 3. Data on the physical, chemical and bacteriological examinations of mud and overlying

\begin{tabular}{|c|c|c|c|c|c|c|c|c|}
\hline Date & Stn. & $\begin{array}{c}\text { Temperature }{ }^{a} \\
\left({ }^{\circ} \mathrm{C}\right)\end{array}$ & $\begin{array}{c}\text { Chlorinity } \\
(\% 0)\end{array}$ & $\begin{array}{c}\text { Inorganic } \\
\text { phosphate }^{b} \\
(\mu \mathrm{mol} / l)\end{array}$ & $\begin{array}{l}\text { Nitrate }{ }^{b} \\
(\mu \mathrm{mol} / l)\end{array}$ & $\begin{array}{l}\text { Nitrite }^{b} \\
(\mu \mathrm{mol} / l)\end{array}$ & $\begin{array}{c}\operatorname{Ammonia}^{b} \\
(\mu \mathrm{mol} / l)\end{array}$ & $\begin{array}{c}\text { Total } \\
\text { nitrogen }^{b} \\
(\mu \mathrm{mol} / l)\end{array}$ \\
\hline \multirow{7}{*}{$\begin{array}{l}\text { Feb 26, } \\
1987\end{array}$} & 1 & 16.5 & 0.3 & 1.2 & 11.0 & 0.3 & 9.0 & 126 \\
\hline & 3 & 17.0 & 3.0 & 1.7 & 8.8 & 0.5 & 6.6 & 130 \\
\hline & 5 & 17.0 & 8.5 & 3.2 & 1.1 & 0.2 & 6.8 & 140 \\
\hline & 7 & 17.0 & 17.3 & 3.0 & 1.7 & 0.1 & 5.6 & 144 \\
\hline & 9 & 17.0 & 18.2 & 2.1 & 1.4 & 0.1 & 5.1 & 135 \\
\hline & 11 & 17.5 & 18.9 & 1.8 & 1.8 & 0.1 & 4.9 & 130 \\
\hline & 13 & 17.5 & 19.3 & 1.2 & 1.9 & 0.1 & 4.9 & 133 \\
\hline \multirow{7}{*}{$\begin{array}{l}\text { Aug 27, } \\
1987\end{array}$} & 1 & 29.5 & 2.5 & 4.2 . & 2.8 & 0.2 & 14.3 & 143 \\
\hline & 3 & 30.0 & 5.4 & 3.3 & 2.1 & 0.2 & 10.7 & 156 \\
\hline & 5 & 29.8 & 14.4 & 7.1 & 1.9 & 0.1 & 9.3 & 165 \\
\hline & 7 & 30.0 & 18.3 & 7.1 & 1.6 & 0.1 & 8.1 & 163 \\
\hline & 9 & 29.8 & 18.3 & 4.7 & 1.5 & 0.1 & 7.4 & 140 \\
\hline & 11 & 29.9 & 18.5 & 4.5 & 1.4 & 0.1 & 6.9 & 138 \\
\hline & 13 & 30.0 & 19.0 & 4.3 & 1.4 & 0.1 & 6.0 & 137 \\
\hline
\end{tabular}

${ }^{a}$, determined by using overlying water (The same as in Table 1);

${ }^{b}$, determined by using pore water. 
relationships between the variables related to water of Nakama River estuary

\begin{tabular}{l|l|l|l|l|l|l|l}
\hline $\mathrm{x}_{7}$ & $\mathrm{x}_{8}$ & $\mathrm{x}_{9}$ & $\mathrm{x}_{10}$ & $\mathrm{x}_{11}$ & $\mathrm{x}_{12}$ & $\mathrm{x}_{13}$ & $\log Y_{w}$ \\
\hline & & & & & & & \\
& & & & & & & \\
& & & & & & & \\
& & & & & & & \\
1.000 & & & & & & & \\
$0.549^{a}$ & 1.000 & & & & & \\
$0.594^{a}$ & $0.625^{a}$ & 1.000 & & & & \\
0.191 & 0.332 & $0.443^{a}$ & 1.000 & & & \\
$0.425^{a}$ & $0.588^{a}$ & 0.320 & 0.191 & 1.000 & & \\
$0.429^{a}$ & $0.691^{a}$ & $0.541^{a}$ & $0.385^{a}$ & $0.907^{a}$ & 1.000 & & \\
$0.494^{a}$ & $0.577^{a}$ & $0.669^{a}$ & 0.359 & $0.698^{a}$ & $0.770^{a}$ & 1.000 & \\
$0.543^{a}$ & $0.742^{a}$ & $0.681^{a}$ & $0.412^{a}$ & $0.560^{a}$ & $0.707^{a}$ & $0.661^{a}$ & 1.000 \\
\hline
\end{tabular}

levels of water bacteria, chlorophyll, particulate carbohydrates and particulate amino acids. This trend strongly suggested that the numbers of water

water from Nakama River estuary

\begin{tabular}{c|c|c}
\hline $\begin{array}{c}\text { Total } \\
\text { carbohydrates } \\
\text { (as mg glucose } \\
\text { /g dry mud) }\end{array}$ & $\begin{array}{c}\text { Total } \\
\text { amino acids } \\
\text { (as mg glutamic } \\
\text { acid/g dry mud) }\end{array}$ & $\begin{array}{c}\text { Mud } \\
\text { bacteria } \\
\text { (cfu/g } \\
\text { wet mud) }\end{array}$ \\
\hline 0.13 & 1.1 & $1.7 \times 10^{6}$ \\
0.09 & 0.5 & $1.7 \times 10^{6}$ \\
0.11 & 0.9 & $2.0 \times 10^{6}$ \\
0.42 & 2.4 & $2.4 \times 10^{6}$ \\
0.12 & 0.8 & $9.0 \times 10^{5}$ \\
0.06 & 0.7 & $5.0 \times 10^{5}$ \\
0.06 & 0.8 & $9.0 \times 10^{5}$ \\
\hline 0.07 & 0.7 & $7.1 \times 10^{6}$ \\
0.54 & 1.6 & $9.5 \times 10^{6}$ \\
0.98 & 2.7 & $1.8 \times 10^{7}$ \\
0.57 & 2.0 & $2.2 \times 10^{7}$ \\
0.44 & 1.1 & $5.8 \times 10^{6}$ \\
0.15 & 0.8 & $9.1 \times 10^{5}$ \\
0.16 & 0.8 & $5.5 \times 10^{5}$ \\
\hline
\end{tabular}

bacteria had a close correlation with the biomass of phytoplankton. Similar tendencies have been confirmed by many other workers (e.g., Straskrabova and Komerkova, 1979; Pedros-Alio and Brock; 1982; Bird and Kalff, 1984; Nagata, 1984). That is, the growth of water bacteria may be supported by the pool of organic matter derived from both living and dead phytoplankton. In addition, the growth of water bacteria seemed to be controlled by the pool of particulate organic matter originating probably from mangrove litter. Detailed discussion on this problem will be described elsewhere.

Bacterial population, most of the nitrogen components and all of the particulate components in the water increased with increasing temperature (Table 2). However, direct coupling between bacterial population and temperature was thought to be doubtful in marine environments (ZoBell, 1946). In this light, it seems reasonable to consider that the seasonality of bacterial population in the water is also brought about indirectly by changes in the phytoplankton biomass.

The possible explanations for the close relationships between bacterial numbers and inorganic 
Table 4. Correlation matrix showing degree of linear relationships between variables related to mud

\begin{tabular}{l|c|c|c|c|c|c|c|c|c|c}
\hline \multicolumn{1}{c|}{ Variables } & $\mathrm{x}_{1}^{\prime}$ & $\mathrm{x}_{2}^{\prime}$ & $\mathrm{x}_{3}^{\prime}$ & $\mathrm{x}_{4}^{\prime}$ & $\mathrm{x}_{5}^{\prime}$ & $\mathrm{x}_{6}^{\prime}$ & $\mathrm{x}_{7}^{\prime}$ & $\mathrm{x}_{8}^{\prime}$ & $\mathrm{x}_{9} \min ^{\prime}$ & $\log Y_{m}$ \\
\hline Temperature $\left(\mathrm{x}_{1}^{\prime}\right)^{a}$ & 1.000 & & & & & & & & & \\
Chlorinity $\left(\mathrm{x}_{2}^{\prime}\right)^{a}$ & 0.139 & 1.000 & & & & & & & \\
Inorganic phosphate $\left(\mathrm{x}_{3}^{\prime}\right)^{b}$ & $0.805^{c}$ & 0.270 & 1.000 & & & & & & \\
Nitrate $\left(\mathrm{x}_{4}^{\prime}\right)^{b}$ & 0.392 & $-0.715^{c}$ & -0.453 & 1.000 & & & & & & \\
Nitrite $\left(\mathrm{x}_{5}^{\prime}\right)^{b}$ & 0.340 & $-0.805^{c}$ & -0.410 & $0.822^{c}$ & 1.000 & & & & & \\
Ammonia $\left(\mathrm{x}_{6}^{\prime}\right)^{b}$ & 0.537 & -0.652 & 0.364 & 0.164 & 0.212 & 1.000 & & & & \\
Total nitrogen $\left(\mathrm{x}_{7}^{\prime}\right)^{b}$ & 0.615 & 0.122 & $0.844^{c}$ & -0.432 & -0.340 & 0.425 & 1.000 & & & \\
Total carbohydrates $\left(\mathrm{x}_{8}^{\prime}\right)$ & 0.520 & 0.147 & $0.743^{c}$ & -0.243 & -0.285 & 0.259 & $0.877^{c}$ & 1.000 & & \\
Total amino acids $\left(\mathrm{x}_{9}^{\prime}\right)$ & 0.265 & 0.162 & 0.593 & -0.211 & -0.335 & 0.153 & $0.792^{c}$ & $0.896^{c}$ & 1.000 & \\
Mud bacteria $\left(\log Y_{m}\right)$ & 0.552 & -0.240 & $0.708^{c}$ & -0.082 & -0.014 & $0.662^{c}$ & $0.853^{c}$ & $0.782^{c}$ & $0.670^{c}$ & 1.000 \\
\hline
\end{tabular}

${ }^{a}$ and ${ }^{b}$, the same as in Table $3 ;{ }^{c}$, significant at $0.01 \%$ level.

nitrogen variables are as follows: the inorganic components may control bacterial growth indirectly by changes in the phytoplankton biomass, or the inorganic nitrogen components may be produced from organic nitrogen compounds by the catalytic action of bacteria, or both.

In view of geographical distribution, the levels of several variables such as chlorophyll and particulate amino acids in the midestuarine water were higher than those in the lower and upper estuaries, i.e. the midestuarine peaks of these variables were observed. These phenomena can be ascribed to the prolonged residence of water in the estuary which causes the accumulation of dissolved nutri-

Table 5. Relevant correlation coefficients between the variables related to the water and the mud from Nakama River estuary

\begin{tabular}{|c|c|c|c|c|c|c|c|c|c|}
\hline \multirow{2}{*}{\multicolumn{2}{|c|}{ Variables }} & \multicolumn{8}{|c|}{ Mud-related variables } \\
\hline & & Inorganic & Nitrate & Nitrita & Ammoni & Total & Total & Total & Mud \\
\hline \multirow{10}{*}{$\begin{array}{l}\text { Water- } \\
\text { related } \\
\text { variables }\end{array}$} & $\begin{array}{l}\text { Inorganic } \\
\text { phosphate }\end{array}$ & 0.002 & -0.288 & -0.157 & -0.219 & 0.224 & 0.287 & 0.486 & 0.174 \\
\hline & $\begin{array}{l}\text { Total } \\
\text { phosphorous }\end{array}$ & 0.350 & -0.231 & 0.018 & $0.711^{a}$ & 0.502 & 0.300 & 0.294 & 0.486 \\
\hline & Nitrate & -0.051 & 0.478 & $0.672^{a}$ & $0.728^{a}$ & 0.240 & 0.255 & 0.209 & 0.421 \\
\hline & Nitrite & 0.441 & -0.100 & 0.110 & $0.796^{a}$ & $0.556^{b}$ & 0.471 & 0.362 & $0.532^{\circ}$ \\
\hline & Ammonia & 0.471 & -0.083 & 0.164 & $0.897^{a}$ & $0.626^{b}$ & 0.506 & 0.329 & $0.626^{b}$ \\
\hline & $\begin{array}{l}\text { Total } \\
\text { nitrogen }\end{array}$ & $0.695^{a}$ & -0.273 & -0.174 & 0.241 & $0.562^{b}$ & 0.321 & 0.272 & 0.255 \\
\hline & $\begin{array}{l}\text { Particulate } \\
\text { carbohydrates }\end{array}$ & 0.226 & 0.024 & $0.533^{b}$ & $0.645^{b}$ & 0.277 & 0.028 & -0.148 & 0.370 \\
\hline & $\begin{array}{l}\text { Particulate } \\
\text { amino acids }\end{array}$ & 0.516 & -0.174 & 0.290 & $0.753^{a}$ & $0.579^{b}$ & 0.372 & 0.127 & $0.559^{b}$ \\
\hline & $\begin{array}{l}\text { Chlorophyll } \\
a\end{array}$ & 0.178 & -0.024 & 0.248 & $0.880^{a}$ & 0.279 & 0.004 & -0.067 & 0.348 \\
\hline & $\begin{array}{l}\text { Water } \\
\text { bacteria }\end{array}$ & $0.569^{b}$ & -0.321 & 0.190 & $0.541^{b}$ & $0.680^{a}$ & 0.520 & 0.292 & 0.512 \\
\hline
\end{tabular}

The basic data for the analysis were given in Tables 1 and 3 .

$a$, significant at $0.01 \%$ level; ${ }^{b}$, significant at $0.05 \%$ level. 
ents released from mud, as suggested by Wright and Coffin (1984). The resulting nutrients may support the growth of phytoplankton.

With the aim to select some important environmental variables for bacterial distribution, the regression analysis was carried out, and the predictive model including 7 environmental variables was obtained. Therefore, water as a bacterial habitat seems to be so diversified that there are no dominant variables controlling bacterial number. In other words, bacterial number in the estuary seems to be controlled by a lot of variables. Considering the $R^{2}$ value of equation 1 , the environmental variables included in the model can explain up to $81 \%$ of the variance of bacterial numbers.

The numbers of mud bacteria were slightly higher in summer than in winter just like that of water bacteria. These tendencies might be attributed mainly to the summer increase of organic matter supplied by the water column. Midestuarine peaks were observed for the levels of mud bacteria, inorganic phosphate, total nitrogen, total carbohydrates and total amino acids in the pore water or mud.

Pravdic (1970) showed that marine sediments were negatively charged in seawater, but most of them were positively charged in freshwater and a reversal of charge occurred between 2 and $6 \% 0$ salinity. Accordingly, particle aggregation due to the neutralization of surface charge should occur in the midestuary, and a relatively large part of the resulting aggregates should settle onto the bottom surface within the midestuary. Naturally, cosedimentation of these aggregates and their attached bacteria should take place, and mud becomes rich in organic nutrients available for bacteria. This may be the reason why midestuarine peak is evident for mud bacteria. Close correlations between the populations of mud bacteria and the concentrations of inorganic components such as phosphate and ammonia can be also explained as follows: these components may control bacterial growth directly, or these components may be produced by the catalytic action of bacteria, or both.

The populations of mud bacteria had close correlations with as many as 5 out of the 9 environmental variables related to mud. Nevertheless, the predictive model for describing the abundance of mud bacteria contained only one variable : total nitrogen concentration in the pore water. This suggests that the total nitrogen concentration is a critical parameter for controlling bacterial density in the mud. Judging from the $R^{2}$ value of equation 2, 73\% of the variance of bacterial populations can be explained by the enviromental variable included in the model.

Exchange of nutrients between mud and overlying water has been studied extensively (e.g., Meyer-Reil et al., 1978; Golterman et al., 1983; Ramohr et al., 1987), but it is not so easy to detect water-mud interaction in the estuary due to tidal current. An attempt was made to evaluate the interaction by computing the degree of correlations between the variables related to water and those related to mud. The population of water bacteria correlated with 3 out of the 8 variables related to mud at the $0.05 \%$ level or better, while the population of mud bacteria correlated with 3 out of the 10 variables related to water at the $0.05 \%$ level (Table $5)$. On the whole, these correlations were not as good as in the foregoing analyses shown in Tables 2 and 4 . This may be because our analysis shown in Table 5 is not sensitive enough to detect such a slow reaction as that catalyzed by the natural microbial populations.

The present study has been designed to analyze the bacterial variables, but the results obtained make it possible to elucidate some of the interrelationships among the enviromental variables. In particular, a broad spectra of interactions were noted for some enviromental variables. This fact strongly suggests that these variables may have great influences on the ecosystem and seem to be richer in ecological information. 


\section{Acknowledgments}

We wish to express our gratitude to Dr. Takatoshi Nishijima of Kochi University for his help in chlorophyll determination. This study was partly supported by a Grant-in-Aid for Enviromental Science from the Ministry of Education, Science and Culture, Japan.

\section{References}

American Public Health Association, 1981. Standard methods for the examination of water and wastewater, 15th ed., The Association, Washington D. C.

Bird, F. and J. Kalff, 1984. Empirical relationships between bacterial abundance and chlorophyll concentration in fresh and marine waters. Can. J. Fish. Aquat. Sci., 41, 1015-1023.

Buck, J.D. and R.C. Cleverdon, 1960. The spread plate as a method for the enumeration of marine bacteria. Limnol. Oceanogr., 5, 78-80.

Golterman, H.L., P.G. Sly and R.L. Thomas, 1983. A study of the Relationship between Water Quality and Sediment Transport. UNESCO, Paris.

Koyama, T., N. Handa. and I. Sugimura, 1972. Kosui kaisui no bunseki (Analyses of lake- and sea-water (in Japanese) Kodan-shia. Tokyo.

Grasshoff, K., M. Ehrhardt and K. Kremling (eds.), 1983. Methods of Seawater Analysis. 2nd edition, Verlag Chemie, Weinheim.

Jones, J.G., 1977. Studies on freshwater bacteria: factors which influence the population and its activities. J. Ecol., 59, 593-613.

Hazen, T.C., 1983. A model for the density of Aeromonas hydrophila in Albemarle Sound, North Carolina. Microbial Ecol., 9, 137-153.

Mahloch, J.L., 1974. Comparative analysis of modeling techniques for coliform organisms in streams. Appl. Microbiol., 27, 340-345.

Meyer-Reil, L.-A., R. Dawson, G. Liebezeit and H. Tiedge, 1978. Fluctuations and interactions of bacterial activity in sandy beach sediments and overlying waters. Mar. Biol., 48, 161-171.

Miyoshi, H. and K. Nakamoto, 1975. Factors influencing bacterial distribution in the sea of Hiuchi-Nada area. Bull. Jap. Soc. Sci. Fish., 41, 645-652.

Nagata, T., 1984. Bacterioplankton in lake Biwa: Annual fluctuations of bacterial number and their possible relationships with enviromental variables. Jpn. J. Limnol., 45, 125-133:

Nishino, S.F., 1986. Direct acridine orange counting of bacteria preserved with acidified Lugol iodine. Appl. Environ. Microbiol, 52, 602-604.

Palumbo, A.V. and Ferguson, R.L., 1978. Distribution of suspended bacteria in the Newport River estuary, North Carolina. Estuar. Coast. Mar. Sci., 7, 521-529.

Pedros-Alio, C. and T.D. Brock, 1982. Assessing biomass and production of bacteria in eutrophic lake Mendota, Wisconsin. Appl. Environ. Microbiol., 44, 203-218.

Pravdic, V., 1970. Surface charge characterization of sea sediments. Limnol. Oceanogr., 15, 230-233.

Ramohr, J., E. Walger and B. Zeitzschet (ed.) 1987. Seawater-Sediment Interactions in Coastal Waters. Springer-Verlag, Berlin.

Souwapa, A., H. Miyoshi and Y. Hata, 1989. Characteristics of bacteria isolated from mangrove estuary of Nakama River, Iriomote Island, Okinawa. Nihon Bisebutsu Seitai Gakkaiho, 3, 59 $-66$.

Straskrabova, V. and J. Komerkova, 1979. Seasonal changes of bacterioplankton in a reservoir related to algae. Int. Rev. Gesamten. Hydrobiol., 64, 285- 302.

Väätänen P., 1982. Effects of freshwater outflows on microbial populations in the Tvarminne archipelago, southern Finland. Holarct Ecol., 5, 61 $-66$.

Wright, R.T. and R.B. Coffin, 1984 . Factors affecting bacterioplankton density and productivity in salt marsh estuaries. In : Proceeding of third International Symposium on Microbial Ecology (edited by M.J. Klug and C.A. Reddy) pp. 485-494. American Society for Microbiology, Washington D.C

ZoBell, C.E. 1946. Marine Microbiology. Chronica Botanica Co., Waltham, Mass.

(Received December 9, 1988 - Accepted April 17, 1989) 